\title{
Online Tracking of Migrating and Proliferating Cells Imaged with Phase-Contrast Microscopy
}

\author{
Kang Li ${ }^{1 *}$, Eric D. Miller ${ }^{2}$, Lee E. Weiss ${ }^{3}$, Phil G. Campbell ${ }^{4}$, Takeo Kanade ${ }^{1 *}$ \\ ${ }^{1}$ Department of Electrical and Computer Engineering, ${ }^{2}$ Department of Biomedical Engineering \\ ${ }^{3}$ Robotics Institute, ${ }^{4}$ Institute for Complex Engineered Systems \\ Carnegie Mellon University \\ 5000 Forbes Ave., Pittsburgh, PA 15213 \\ * $\{$ kangli, tk $\}$ ecs. cmu. edu
}

\begin{abstract}
Automated visual-tracking of cell population in vitro using phase-contrast time-lapse microscopy is vital for the quantitative and systematic study of cell behaviors, including spatiotemporal quantification of migration, proliferation, and apoptosis. The low image quality, high and varying density of the cell culture, and the complexity of cell behaviors pose many challenges to existing tracking techniques. This paper presents a fully-automated multitarget tracking system that can simultaneously track hundreds of cells and efficiently cope with these challenges. The approach exploits a fast topology-constrained level-set method in conjunction with a stochastic motion filter, with a careful formulation that makes it suitable for real-time tracking during acquisition. Our methodology was applied to human tissue cell tracking in vitro under various imaging conditions and yielded a $88.4 \%$ tracking accuracy.
\end{abstract}

\section{Introduction}

Biological discovery is advancing toward the use of combinatorial, high-throughput experimental approaches for applications in genomics, proteomics, drug development, tissue engineering and stem cell research. Computer-aided bioprinting $[22,2,13]$ is one such approach, which can programmatically create complex patterned arrays of extracellular components, including hormones (e.g. growth factors) and extracellular matrix molecules, for inducing and directing cell fates. Cells can respond to these printed patterns in multiple ways including: migration (translocation), proliferation (through a division mechanism called mitosis), differentiation (by which cells acquire more specialized functions), quiescence (inactivity) and apoptosis (death). The study of how the patterns regulate migration, proliferation, and apoptosis requires the use of non-fluorescent phase-
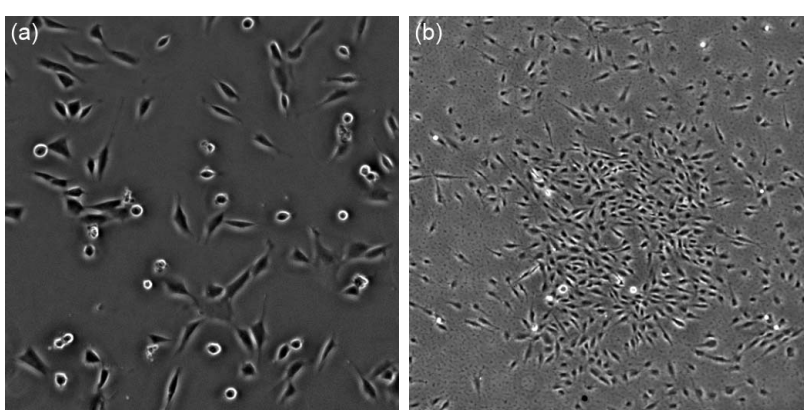

Figure 1. Phase-contrast microscopy images of cell populations

contrast microscopy to record the cellular responses over an extended period of time (5-10 days), which routinely produces extremely large datasets with low signal-to-noise ratios (Figure 1). Typical experiments produce over 100 gigabytes (GB) of image data consisting of about 40000 frames, with up to thousands of cells in each frame. This makes $a u$ tomated tracking and analysis of cells critical in efficiently studying the underlying biological mechanisms. However, the high processing demand, the varying density of the cell culture (with cells dividing/dying, leaving/reentering the field-of-view), and the complexity of the cellular topologies (shape deformation, close contact, and partial overlap) pose many challenges to existing tracking techniques.

In this paper, we present a multi-target tracking system that can simultaneously track hundreds to thousands of cells, and can successfully cope with the aforementioned challenges. The system exploits a two-level design. The lower level consists of several modules including: a cell detector, a fast topology-constrained level-set cell tracker, and a stochastic motion filter (e.g. Kalman filter [9]). The higher level comprises a reasoning unit called a track arbitrator, which fuses and coordinates the outputs of the lower-level components. The efficiency and causal formulation of the 
method makes it suitable for high-throughput online processing for tracking during image acquisition.

\subsection{Previous Work on Object Tracking}

The high processing demand for extended-time studies of large cell populations rules out the use of manual or computer-aided interactive tracking. Fully-automated techniques are required. Methods for automated object tracking mainly involve two different approaches: tracking by detection and tracking by model-evolution.

The first approach performs object detection and interframe data association in two independent stages. This approach is effective when the objects are well-separated, but faces ambiguousness if the objects undergo close contact, and when the detector produces split/merged measurements. Recently, Khan et al tackled the problem of object contact [11] by incorporating a Markov random field prior distribution into the particle filter [6] framework, for modeling object interactions and maintaining object identities. The same authors addressed the issue of split/merged measurements [12], but under the assumption that the number of objects is fixed, which is violated in our problem. Furthermore, neither work has considered the scenario where the object can divide and replicate itself.

The second approach involves the creation of mathematical models, either appearance or shape models, which are fitted to the objects and are evolved over time to follow the object movements. This category encompasses a large spectrum of techniques with varied capabilities. The parametric active contours (e.g, snakes [10]) and mean-shift [4] models have been explored in the past for tracking multiple migrating cells under phase-contrast microscopy [24, 5].

The classic snake model has two major drawbacks for cell tracking. One is its inability to handle topology changes of the contour, which is crucial for modeling cell division. The other is its catastrophic behavior when the cells are in close contact, where the contour associated with one cell may easily be attracted to a neighboring cell. Zimmer et al $[24,25]$ extended the snake model using the ideas of adding repulsive forces between snakes to handle close contact of cells, and incorporating "topological operators" to handle cell division. Both extensions, however, significantly increased the computational overhead, making the method prohibitively expensive for tracking a large number of cells.

Debeir et al [5] considered a somewhat simplified problem of tracking only the centroid positions, but not the boundaries, of the cells, which permits a mean-shift-based model [4] to be used. However, similar to the snakes, this model cannot handle cell division. As a remedy, the authors proposed to track backwards (from the last frame to the first), which simplified the problem but made the tracking noncausal and unsuitable for online processing. Moreover, this tracker requires manual initialization, and cannot auto- matically incorporate new cells entering the field of view.

Interestingly, cell tracking using the level-set methodology [15], which is increasingly deemed a more powerful framework for image analysis than its parametric counterparts, has been dismissed in several previous reports $[24,25,5,1]$. One criticism has been against the topological flexibility (i.e., the freedom to merge and break) of level-set represented object boundaries. This flexibility, while permitting efficient modeling of cell division, does not prevent two contacting boundaries from merging. Cell fusion, however, is impossible in most biological contexts, including ours. In fact, previous studies on level-set cell-tracking either did not consider contacting cells [14], or resorted to off-line post-processing to correct cell fusions [23]. Another criticism of the level-set method is its high computational expenses. However, as we will demonstrate, both disadvantages can be overcome by taking advantage of recent developments on level-set methods, including topology control and fast implementations. Indeed, the potential of the level-set model for tracking has yet to be fully realized. While the topology control of level sets has been studied more extensively for image segmentation [7, 19], its potential for tracking is under-explored.

\section{Methods}

Our tracking system consists of four major components: 1) Cell detector, which detects and labels candidate cell regions in the input image based upon adaptive segmentation; 2) Model tracker, which, by exploiting a novel fast topology-constrained level-set formulation, tracks cell regions across frames following the image cues and the motion predictions; 3) Motion filter, which provides temporal contexts to the cell tracker and track arbitrator; and 4) Track arbitrator, which manages the whole tracking task by adding newly-entered cells to the record, removing departed/dead cells, re-labeling divided cells, and recovering lost tracks, by fusing the outputs from the first three modules using high-level reasoning based on what is and what is not physically possible. All components work collaboratively to produce the final spatiotemporal history of cell trajectories, including centroid positions, boundaries, migration velocities, mean intensities, and parent-daughter linkage for all cells in an experiment.

\subsection{Cell Detection}

Cells in phase-contrast images normally appear as dark regions surrounded by brighter halo artifacts, except for mitotic (dividing) or apoptotic (dying) cells, which are smaller, rounder and have very bright appearances.

The cell detector works by classifying the image pixels into cell (C) and background (B) classes. The output is a binary map of cell regions, denoted $\zeta^{k}$, where $k=1, \ldots, K$ 
is the frame index. Each connected foreground component in $\zeta^{k}$ is considered as a cell candidate in frame $k$. The classification is based on the maximum a posteriori principle:

$c_{\text {MAP }}(\mathbf{x})=\underset{c}{\arg \max } p(c \mid I(\mathbf{x}))=\underset{c}{\arg \max } p(I(\mathbf{x}) \mid c) p(c)$,

where $c \in\{C, B\}$ denotes the pixel class, $\mathbf{x}=(x, y)$ is the pixel location, and $I(\mathbf{x})$ is the pixel intensity. Given the (normalized) cell histogram $\left(h_{C}\right)$ and background histogram $\left(h_{B}\right)$ (as derived below), the classifier can be implemented as:

$$
c_{M A P}(\mathbf{x})= \begin{cases}C, & h_{C}(I(\mathbf{x})) \geq h_{B}(I(\mathbf{x})), \\ B, & \text { otherwise }\end{cases}
$$

The mitotic/apoptotic cells and the non-mitotic nonapoptotic cells are detected separately using two sets of histograms. The appearances of the mitotic/apoptotic cells are usually very distinctive. Their histograms are constructed by off-line learning utilizing a small amount of training data. On the other hand, the histograms for the non-mitotic non-apoptotic cells exhibit more fluctuations across different frames or datasets. Therefore, starting from the pretrained histograms $h_{C}^{0}$ and $h_{B}^{0}$, the histograms are updated for each frame using the following auto-regression:

$$
h_{c}^{k}=(1-\alpha) h_{c}^{k-1}+\alpha \hat{h}_{c}^{k}, \quad c \in\{C, B\}, 0 \leq \alpha \leq 1 .
$$

The histograms $\hat{h}_{c}^{k}$ are constructed based on a rough segmentation of the cells in the current frame using background subtraction followed by Otsu thresholding [16]. Under the assumption that the background is piecewise linear, the background is estimated using a "rolling-ball" algorithm [21], which simulates rolling a certain-sized ball over the image plane, filling in the potholes that matches the ball size.

\subsection{Level-Set Cell Tracker}

Let $\Omega \subset \mathbb{R}^{2}$ denote the image domain. The level-set method represents any contour $\Gamma^{k} \subset \Omega$ as the zero level set of a scalar function $\phi^{k}(\mathbf{x}): \Omega \rightarrow \mathbb{R}$ (dubbed the level-set

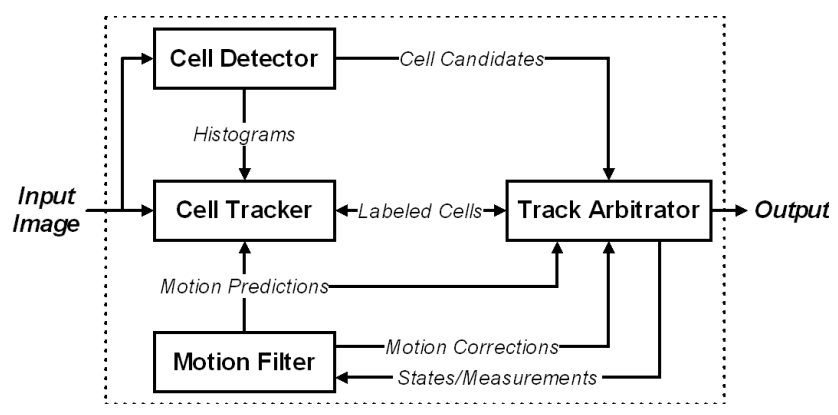

Figure 2. System Overview function), i.e., $\Gamma^{k}=\left\{\mathbf{x} \mid \phi^{k}(\mathbf{x})=0\right\}$. For cell tracking, the contour $\Gamma^{k}$ consists of the cell boundaries in frame $k$.

Let $\mathbf{N}^{k-1}$ be the set of cell labels that exist in frame $k-1$. We use $\Omega_{0} \subset \Omega$ to denote the background, and $\Omega_{n} \subset \Omega\left(n \in \mathbf{N}^{k-1}\right)$ to denote the cell regions. Let $\Omega_{C}=\bigcup_{n \in \mathbf{N}^{k-1}} \Omega_{n}$. The level set function for frame $k$ is initialized to satisfy:

$$
\phi^{k}(\mathbf{x})= \begin{cases}<0, & \text { if } \mathbf{x} \in \Omega_{C}, \\ =0, & \text { if } \mathbf{x} \in \Gamma^{k-1}, \\ >0, & \text { if } \mathbf{x} \in \Omega_{0} .\end{cases}
$$

To keep track of the labeled cell regions, another auxiliary region labeling function $\psi^{k}$ is evolved simultaneously with $\phi^{k}$, which is initialized by

$$
\psi^{k}(\mathbf{x})=n, \quad \text { if } \mathbf{x} \in \Omega_{n}, \quad\left(n \in\{0\} \cup \mathbf{N}^{k-1}\right) .
$$

$\psi^{k}$ is also the output of the level-set tracker, which will carry labels of the propagated cell regions for frame $k$.

Intuitively, the placement of each cell boundary should not only match the cell appearance in the current frame, but also be a consequence of a "conceivable" movement following the cell motion pattern. Mathematically, $\phi^{k}$ is evolved to minimize an energy functional that is the weighted combination of three terms that are derived from: 1) the pixel intensities $\left.\left(E_{\text {region }}\right) ; 2\right)$ image gradient magnitudes $\left(E_{\text {edge }}\right)$; and 3$)$ the cell motion predictions $\left(E_{\text {motion }}\right)$ :

$$
E=E_{\text {region }}+\mu E_{\text {edge }}+\nu E_{\text {motion }},
$$

with

$$
\begin{aligned}
E_{\text {region }} & =\sum_{n \in\{0\} \cup \mathbf{N}^{k-1}} \int_{\Omega_{n}}-\log p\left(I(\mathbf{x}) \mid \Omega_{n}\right) d \mathbf{x}, \\
E_{\text {edge }} & =\sum_{n \in \mathbf{N}^{k-1}} \int_{\Omega_{n}} g(\mathbf{x}) \delta\left(\phi^{k}(\mathbf{x})\right)\left|\nabla \phi^{k}(\mathbf{x})\right| d \mathbf{x}, \\
E_{\text {motion }} & =\sum_{n \in \mathbf{N}^{k-1}} \int_{\Omega_{n}}\left(v-\log p\left(I(\mathbf{x}), \mathbf{x} \mid \hat{\mathbf{s}}_{n}^{k \mid k-1}\right)\right) d \mathbf{x} .
\end{aligned}
$$

Here, $\mu, \nu$ and $v$ are real coefficients. $\delta(\cdot)$ is the Dirac delta function, and $g(\cdot)$ is an edge indicator function that takes on small values near image edges. The probability function $p\left(I(\mathbf{x}) \mid \Omega_{n}\right)$ is the pixel intensity distribution for each region, and $p\left(I(\mathbf{x}), \mathbf{x} \mid \hat{\mathbf{s}}_{n}^{k \mid k-1}\right)$ is the probability that the pixel $\mathbf{x}$ and its intensity match the prediction of the motion filter (Section 2.3). Both distributions are assumed to be independent at each pixel.

The effect of minimizing $E$ is as follows. The region energy term $E_{\text {region }}$ encourages the cell/background configuration to match the image appearance. The edge energy $E_{\text {edge }}$, also known as the geodesic energy [3], draws the contour to high gradient magnitude (i.e., edge) locations. Finally, the motion term $E_{\text {motion }}$ attracts the contour to the modes of the predicted cell centroid distributions. 


\subsubsection{Cell Merging Prevention by Topology Control}

To prevent cell fusion, it is important to introduce topology constraints that permit division but prohibit merging. To achieve this, we borrow the concept of topological numbers from digital topology [7]. Let $N_{8}(\mathbf{x})$ be the set of 8 neighbors of pixel $\mathbf{x}$. The topological number of $\mathbf{x}$ with respect to $\Omega_{C}$, denoted $T_{C}(\mathbf{x})$, is the number of 4-connected components in the set $\Omega_{C} \cap N_{8}(\mathbf{x})$. Similarly, the topological number of $\mathrm{x}$ with respect to the background $\Omega_{0}$, denoted $T_{0}(\mathbf{x})$, is the number of 8-connected components in the set $\Omega_{0} \cap N_{8}(\mathbf{x})$. Let $o(\mathbf{x})$ denote the number of cell regions that overlap with $N_{8}(\mathbf{x})$. Then, the relaxed topological number [20] for pixel $\mathbf{x}$ is defined as:

$$
T_{r}(\mathbf{x})=\min \left(o(\mathbf{x}), \max \left(T_{C}(\mathbf{x}), T_{0}(\mathbf{x})\right)\right) .
$$

The boundaries of two different cell regions $\Omega_{i}, \Omega_{j}$ can merge only if the level set function changes sign from positive to negative at a point $\mathbf{x}$ with $T_{r}(\mathbf{x})>1$. By detecting the points where $T_{r}>1$, and preventing the level set function from changing sign at these points, we can effectively prevent different cell regions from merging.

\subsubsection{Implementation}

Instead of minimizing (1) by numerically evaluating its first variation, we adopt the subsecond-speed algorithm proposed by Shi et al in [20]. This algorithm is limited to pixel-level accuracies. However, this is deemed adequate for our problem, since our primary goal is to track the trajectories of cells over time, rather than to delineate the cell boundaries at a subpixel accuracy. A pixel-level accuracy is also sufficient for extracting all features required to assist the tracking and analysis.

\subsection{Stochastic Motion Filtering}

A motion filter is the fundamental building block of many tracking systems, which provides recursive estimations of the target states (position, speed and/or number) based on noisy measurements. Two of the best-known filters are the Kalman filter [9] and the particle filter [6].

In our case, the target state vector $\mathbf{s}_{n}^{k}$ of the $n$-th cell in frame $k$ consists of the cell centroid position $\left(x_{n}^{k}, y_{n}^{k}\right)$, migration speed $\left(\dot{x}_{n}^{k}, \dot{y}_{n}^{k}\right)$, and the mean pixel intensity, $i_{n}^{k}$, of the cell region: $\mathbf{s}_{n}^{k}=\left(i_{n}^{k}, x_{n}^{k}, y_{n}^{k}, \dot{x}_{n}^{k}, \dot{y}_{n}^{k}\right)^{T}$. The measurement vector, denoted $\mathbf{z}_{n}^{k}$, consists of the computed centroid and mean intensity of the set of pixels with label $n$ in the level-set tracker output $\psi^{k}$.

For each cell, we estimate its state $\mathbf{s}_{n}^{k}$ based on the sequence of all available measurements up to time $k$. The estimation is done in two stages: prediction and correction. The former stage makes a prediction, $\hat{\mathbf{s}}_{n}^{k \mid k-1}$, of the state at time $k$ based on the state history up to time $k-1$; the latter stage generates a refined estimate $\hat{\mathbf{s}}_{n}^{k \mid k}$, by incorporating the newly-arrived measurement $\mathbf{z}_{n}^{k}$. Essential to any motion filter are a motion model that describes the target dynamics, and a measurement model that relates states to measurements. Before probing further into the formulation of these two models, we need some understanding of the physical process behind cell motility.

\subsubsection{Physical Process of Cell Motion}

The mechanisms behind cellular motion are far from being fully understood. However, it is generally believed that the migratory motion of a cell consists of two components: a directed motion in response to the spatial variations of the underlying physicochemical environment, and a random motion due to the stochastic nature of the membrane adhesions and cytoskeleton contraction events [18]. Moreover, cell migration is found to occur by a sequence of events: 1) membrane extension; 2) formation of new adhesion sites at the forward edge; 3 ) accumulation of tension in the cell; and 4) release of adhesion sites at the trailing edge [18]. The membrane extension event is among the most visible in images. It causes a migrating cell to appear elongated, with its long axis in alignment with the migration direction, a phenomenon referred to as cell polarization.

\subsubsection{Computer Realization}

Based on our current (limited) knowledge of cell motion, we construct the motion model and measurement model under a linear Gaussian assumption. We use a second-order autoregressive process, where the states at a given time depend on two previous time steps. Let $\mathbf{S}_{n}^{k}=\left(\mathbf{s}_{n}^{k}, \mathbf{s}_{n}^{k-1}, \overline{\mathbf{s}}_{n}\right)^{T}$, where $\overline{\mathbf{s}}_{n}$ is the mean state of cell $n$. We define:

$$
\begin{aligned}
\text { Motion model: } & \mathbf{S}_{n}^{k}=\mathbf{A}_{n} \mathbf{S}_{n}^{k-1}+\mathbf{B}_{n} \mathbf{v}_{n}^{k-1}, \\
\text { Measurement model: } & \mathbf{z}_{n}^{k}=\mathbf{H} \mathbf{S}_{n}^{k}+\mathbf{w}_{n}^{k},
\end{aligned}
$$

The matrices $\mathbf{A}_{n}, \mathbf{B}_{n}$ and the mean state $\overline{\mathbf{s}}_{n}$ are initialized using off-line estimated parameters, and updated online every $K_{s}$ (where $K_{s}$ is a constant) frames by learning from the tracking history of each cell in the previous $K_{s}$ frames. The learning methodology is adopted from [17]. The matrix $\mathbf{H}$ equals $\left(\mathbf{I}_{3 \times 3} \mathbf{0}_{3 \times 7}\right)$ with $\mathbf{I}$ being the identity matrix. The vector $\mathbf{v}_{n}^{k-1}$ is a zero-mean Gaussian variable with covariance $\mathbf{Q}=\mathbf{I}_{5 \times 5}$; and the vector $\mathbf{w}_{n}^{k}$ is a time-varying zeromean Gaussian white noise with covariance $\mathbf{R}_{n}^{k}$, computed as the covariance of the set $\left\{I(\mathbf{x}), \mathbf{x} \mid \psi^{k}(\mathbf{x})=n\right\}$, accounting for the cell polarization effect. This formulation allows us to utilize the standard Kalman filter realization [9].

\subsection{Track Arbitration}

The track arbitrator fuses the outputs of the previous subsystems, to determine the final cell trajectories. The arbitra- 
tion procedure (Algorithm 1) is grounded on the fact that the number of cells can change in successive frames only due to four reasons: 1) mitosis; 2) apoptosis; 3) new cells entering from the image border; or 4) cells leaving the image border.

The algorithm first searches for discrepancies between the outputs of the cell detector and tracker, $\zeta^{k}$ and $\psi^{k}$ (Line 1). Any cell candidate in $\zeta^{k}$ that does not correspond to a cell region in $\psi^{k}$ is deemed to arise due to one of the four reasons: 1) it is a new cell; 2) it is a daughter cell of one of the existing cells, but is not properly tracked during mitosis; 3 ) it is a migrating cell that is not properly followed by the tracker; or 4) noise. These cell candidates are added to the set $\boldsymbol{\Omega}_{\text {nolabel }}$ for pending decisions. Then, cell labels are sought that are not propagated from the previous to the current frame (Line 2). This may happen if the corresponding cell regions vanish during the level set evolution. This reflects three possibilities: 1) the cells died and were released into the media; 2) the cells became invisible due to noise; or 3) the contour failed to follow the cells; All the lost labels are added to the set $\mathbf{N}_{\text {lost }}$ for pending decisions.

Meanwhile, mitosis and apoptosis events are being detected (Lines 3,4). Cells undergoing mitosis/apoptosis typically exhibit a series of morphological and appearance changes $[5,23]$. These include an initial decrease of cell area, increase of circularity, and increase of intensity. For mitosis, these are followed by a reverse process and the cell eventually divides. For apoptosis, the reverse process will not occur and the cell will shrink to a small lifeless bright dot or disappear. The information is used to compute two confidence scores. A mitotic or apoptotic event is detected if the corresponding score exceeds a preset threshold.

Next, a pairing between the unlabeled cell candidates in $\boldsymbol{\Omega}_{\text {nolabel }}$ and the lost labels in $\mathbf{N}_{\text {lost }}$ is attempted (Line 5). The pairing is according to a cost function that considers the distance and the intensity difference between the cell candidate and the lost cells in their predicted states. We use a bipartite graph matching algorithm [8] with robust outlier handling to minimize this cost function and obtain a globally optimal pairing. Not all cell candidates will find their matches and vice versa. This is either because the cell candidate is an outlier (due to noise), or because the lost cell is dead or has left the field-of-view. These cases are handled at the end of the arbitration process (Lines 6,8).

Whenever the track arbitrator makes a decision, it performs one of the following operations on the forest of cell trajectories: AddTree, AddBranch (to a tree), UpdateBranch, or Terminate (a branch). For the new cells, new state vectors and the corresponding motion filter parameters are initialized. For non-terminated tracks, measurements are computed from labeled cell regions and are fed back to the motion filter. Note that after apoptosis is detected, the corresponding cell branch may not be immediately terminated (Line 4). This is because the remains of the dead cell

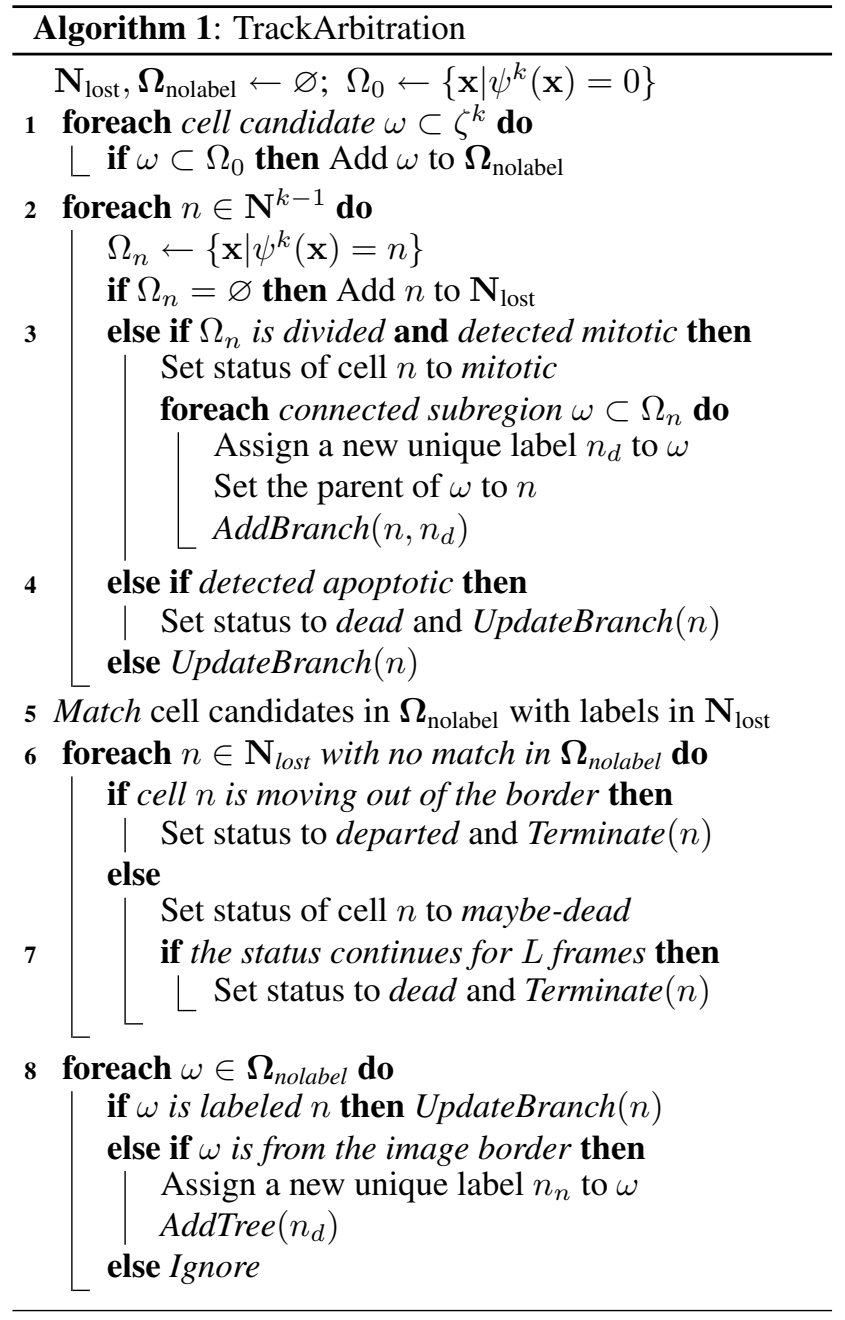

may still be visible in the images, which will be tracked until they become invisible. In addition, a delayed-termination mechanism is used to guard against temporary disappearance of cells (Line 7). The delay parameter $L$ is typically set to 2 to 5 frames, depending on the acquisition interval.

\section{Experiments and Results}

The tracking system is implemented in MATLAB, with the cell detector and level-set tracker written as MATLAB external libraries in $\mathrm{C}++$. The experimental environment closely simulates online processing scenarios. The inputs to the system are illumination-normalized gray-scale images generated by the acquisition software. The illumination normalization is done by dividing each input frame by a smoothed copy of itself.

\subsection{Data}

The experiments utilized 5 uncompressed image sequences of human MG-63 osteosarcoma cells cultured un- 
der standard conditions $\left(37^{\circ} \mathrm{C}, 5 \% \mathrm{CO}_{2}\right)$. The sequences are categorized into two sets ( $\mathrm{A}$ and $\mathrm{B}$ ), according to the imaging protocol and how the cells are seeded.

Data Set $A$ includes 4 image sequences acquired with a 12-bit Qimaging Retiga EXi Fast 1394 CCD camera mounted on a Zeiss Axiovert $135 \mathrm{TV}$ microscope, at a rate of 4 minutes/frame for 10 hours. Each acquired sequence consists of 150 frames, with a frame dimension of $1280 \times 1024$ pixels, and a resolution of $1.9 \mu \mathrm{m} / \mathrm{pixel}$ at a magnification ratio of 4.9:1. The cells are seeded randomly on a polystyrene dish. The images are cropped to a size of $512 \times 512$ pixels (Figure $1(\mathrm{a})$ ) to speed up processing and evaluation. The cell populations in the cropped sequences are in the range of 80-110 cells/frame. One of the four sequences is used for training, the rest is used for testing.

Data Set $B$ includes 1 sequence (Figure 1(b)) recorded by an 8-bit CCD camera on a Zeiss IM35 microscope. The sequence lasts for 43.5 hours with a frame interval of 15 minutes, corresponding to 174 frames/sequence. The frame dimension is $512 \times 512$ pixels with a resolution of 3.9 $\mu \mathrm{m} /$ pixel at 5:1 magnification. The cells are seeded randomly on a fibrin-coated slide, on which a $0.75 \times 0.75 \mathrm{~mm}^{2}$ uniformly-concentrated square pattern of fibroblast growth factor-2 (FGF-2) was created using a bioprinter [22]. The cell population in the sequence is in the range of 350-750 cells/frame. The first 40 frames of the sequence are reserved for training, the rest is used for testing.

\subsection{Initialization}

The initial cell populations are relatively sparse in our experiments, and the tracking is automatically initialized by the cell detector in the first frame of each testing sequence. The first 10 frames of each sequence are considered as "warming-up" frames to allow the system to stabilize. The actual measurements start with the 11th frame (referred to as the initial frame in the sequel). To evaluate the initialization accuracy, we define precision as the ratio of the number of detected cells to the total number of detected objects, and recall as the ratio of the number of detected cells to the total number of cells actually in the image (determined visually by a human observer). Overall, the automatic initialization achieved a precision of $98.1 \%$ and a recall of $96.6 \%$ (measured in the initial frames).

\subsection{Tracking Examples}

Figure 3 demonstrates that the topology-constrained level set can effectively prevent merging of closely contacting cells and maintain cell identities. The images are cropped from a sequence in Data Set A. Figure 4 shows an example of tracking mitotic and apoptotic cells. The images are taken from Data Set B.
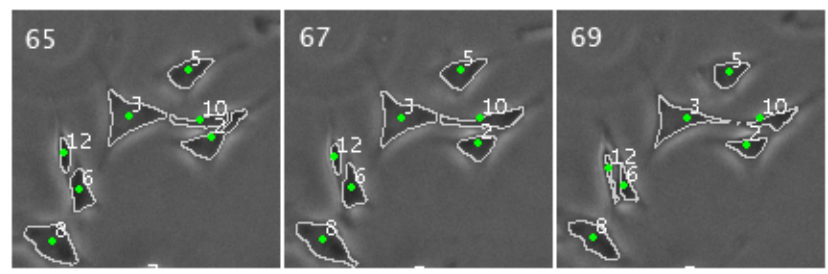

Figure 3. Tracking closely contacting and partially overlapping cells. The numbers at the top-left corner are the frame indices. Cells with labels 2 and 10 are partially overlapping in frames 65-67. Cells 6 and 12 are closely passing each other.
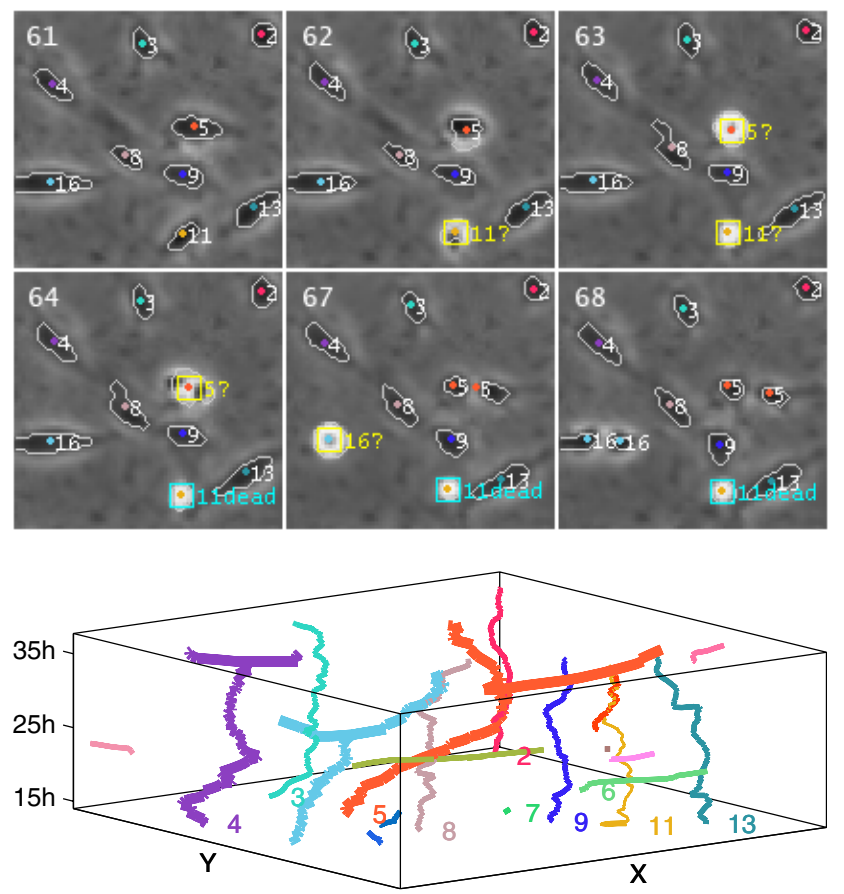

Figure 4. Tracking mitotic and apoptotic cells. Top: six frames of a sequence with cell boundaries and centroids overlaid. Question marks indicate cells in intermediate stages (either mitotic or apoptotic). For daughter cells, the label of their parent is shown. Bottom: a spatial-temporal plot of the corresponding forest of tracks for the image sequence. Branched trees are thickened.

\subsection{Comparison with Manual Tracking}

The centroid locations of the cells for all sequences in Data Set A are manually tracked by a human operator. Only the cells that appear in the initial frame of each sequence and their children are tracked. The manually and automatically tracked trajectories (branches) are paired in the initial frame of each sequence, and are compared in the remaining frames. An automatically tracked cell trajectory is considered valid only if it follows the same cell through all the frames that the cell appears. Our criterion for valid trajectories is more stringent than the distance-based criterion used by Debeir et al [5], since any swapping of identities between two nearby cells will invalidate the trajectories of 
both cells and their children. Finally, for all the valid trajectories, the distances between the manually and automatically placed cell centroids are computed and reported as mean \pm standard deviation. The tracking validity results are shown in Table 1. For the valid cell trajectories, comparison between the automatically tracked and manually placed cell centroid positions shows a difference of $2.4 \pm 2.3$ pixels $(4.6 \pm 4.5 \mu \mathrm{m})$. Since the MG-63 cells are approximately $100 \mu \mathrm{m}$ in size when fully stretched out, the cell centroid positioning difference is much smaller than the cell size.

Table 1. Tracking validity compared to manual tracking

\begin{tabular}{|c|c|c|c|c|}
\hline & A1 & A2 & A3 & Overall \\
\hline Total Trajectories & 81 & 68 & 93 & 242 \\
\hline Valid Trajectories & 70 & 62 & 82 & 214 \\
\hline$\%$ Valid & $86.4 \%$ & $91.2 \%$ & $88.2 \%$ & $88.4 \%$ \\
\hline
\end{tabular}

\subsection{Categorical Analysis of Tracking Errors}

The sequence in Data Set B has a peak cell population of over 700 cells/frame, making manual cell tracking unrealistic. Therefore, the cells are first tracked automatically using the tracking system. The results (Figure 6) are then loaded into a graphical editing tool, and corrected by a human operator until no error is discernable. The operator can correct 7 different types of errors that the tracking system may make, including: miss/false detection of a newly-entered cell (MN/FN), miss/false detection of mitosis (MM/FM), early/late termination of a cell trajectory (ET/LT), or swapping of cell identities (SW). All manual corrections are counted, as listed in Table 2. For comparison, the total numbers of recorded cell trajectories before and after manual corrections are 1425 and 1382, respectively; and the total numbers of mitotic events before and after corrections are 485 and 469, respectively. The relatively high rates of false detection of mitosis and new cells reflect that the system is oversensitive to these events. However, most of the falsely detected cells were automatically removed after a small number of frames. Swapping of cell identities occurred mostly in densely populated regions, where the boundaries between cells are highly blurred.

Table 2. Categorical analysis of tracking errors

\begin{tabular}{lllllllll}
\hline \multirow{2}{*}{ Counts } & $\frac{\text { MN }}{0}$ & $\frac{F N}{12}$ & $\frac{M M}{13}$ & $\frac{F M}{29}$ & $\frac{\text { ET }}{4}$ & $\frac{\text { LT }}{5}$ & $\frac{\text { SW }}{18}$ \\
\hline
\end{tabular}

\subsection{Growth Factor Directed Cell Responses}

Bioprinting and time-lapse image analysis have allowed us to simulate biological patterning of endogenous hormones in laboratory environments, and study its relation to cell responses. However, to date, researchers have primarily been relying on manual or semiautomated image analysis, which usually takes weeks for a single experiment and can achieve little beyond simple cell counting. With our new automated cell tracking methodology, the cell responses can be analyzed in ways that are not previously feasible. The results shown in Figure 5 are extracted from the tracking results of Data Set B, which verify that the cells on-pattern are proliferating and migrating at a higher rate than the cells off-pattern. Moreover, statistics show that the cells are entering the pattern 155 times, while leaving the pattern 143 times. These two comparable numbers further confirm the claim by Miller et al [13] that the primary organizational response of the cells to uniformly-concentrated growth-factor patterns is proliferation and not migration.
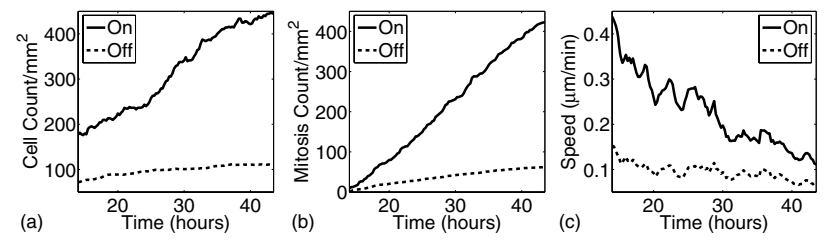

Figure 5. Cell responses on and off the printed FGF-2 growth factor pattern. (a) Cell counts versus time; (b) Accumulated number of mitoses versus time; (c) Average migration speed versus time.

\subsection{Computational Speed}

The tracking system runs at an average speed of 200 frames/hour for processing the images in our experiments, on a workstation with a $3 \mathrm{GHz}$ processor and $4 \mathrm{~GB}$ memory. Since the processing time for each frame (approx. $20 \mathrm{sec}$.) is only a fraction of the typical image acquisition interval, the system is well suited for tracking during acquisition.

\section{Conclusion}

We identified the key components of a fully-automated system capable of simultaneously tracking hundreds of cells under phase-contrast microscopy during acquisition. We illustrated how the different components of the system can be made collaborative to take advantage of both the tracking-by-detection and the tracking-by-model-evolution ideas. We demonstrated that incorporating topological constraints is a key to successful applications of the level set method to cell-tracking. In addition to the reported experiments, satisfactory tracking results were also obtained for other cell types, including Swiss 3T3 cells and stem cells 

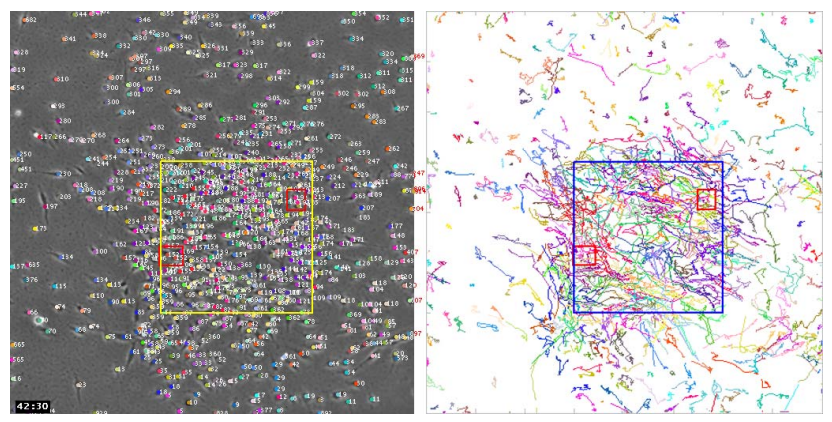

Figure 6. Automatically-tracked cell trajectories. The yellow and blue squares indicate the location of the printed growth-factor pattern. Red rectangles indicate cell division. Cells are displayed using the colors and labels of their farthest ancestors.

(omitted for intellectual property reasons). Our methodology enables automatic quantification of cell migration, proliferation and apoptosis. Extension to quantifying quiescence will be straightforward; and the identification of differentiation can be achieved using cell shape changes or fluorescent biomarkers. Further improvements on robustness and processing speed are currently in progress.

\section{References}

[1] O. Al-Kofahi, R. J. Radke, S. K. Goderie, Q. Shen, S. Temple, and B. Roysam. Automated cell lineage construction: A rapid method to analyze clonal development established with murine neural progenitor cells. Cell Cycle, 5(3):327335, Feb. 2006. 2

[2] P. G. Campbella, E. D. Miller, G. W. Fisher, L. M. Walker, and L. E. Weiss. Engineered spatial patterns of FGF-2 immobilized on fibrin direct cell organization. Biomaterials, 26:6762-6770, Nov. 2005. 1

[3] V. Caselles, R. Kimmel, and G. Sapiro. Geodesic active contours. Int. J. Computer Vision, 22(1):61-79, 1997. 3

[4] D. Comaniciu, V. Ramesh, and P. Meer. Real-time tracking of non-rigid objects using mean shift. In Proc. IEEE Conf. Comp. Vision and Patt. Recog., volume 2, pages 142-149, June 2000. 2

[5] O. Debeir, P. V. Ham, R. Kiss, and C. Decaestecker. Tracking of migrating cells under phase-contrast video microscopy with combined mean-shift processes. IEEE Trans. Med. Imag., 24:697-711, June 2005. 2, 5, 6

[6] N. J. Gordon, D. J. Salmond, and A. F. M. Smith. Novel approach to nonlinear/non-gaussian bayesian state estimation. IEE Proceedings-F, 140(2):107-113, Apr. 1993. 2, 4

[7] X. Han, C. Xu, and J. L. Prince. A topology preserving level set method for geometric deformable models. IEEE Trans. Pattern Anal. Machine Intell., 25(6):755-768, June 2003. 2, 4

[8] R. Jonker and A. Volgenant. A shortest augmenting path algorithm for dense and sparse linear assignment problems. Computing, 38:325-340, 1987. 5
[9] E. Kalman, Rudolph. A new approach to linear filtering and prediction problems. Transactions of the ASME - Journal of Basic Engineering, 82(Series D):35-45, 1960. 1, 4

[10] M. Kass, A. Witkin, and D. Terzopoulos. Snakes: Active contour models. Int. J. Computer Vision, pages 321-331, 1988. 2

[11] Z. Khan, T. Balch, and F. Dellaert. An MCMC-based particle filter for tracking multiple interacting targets. In Proc. European Conf. Comp. Vision, volume IV, pages 279-290, 2004. 2

[12] Z. Khan, T. Balch, and F. Dellaert. Multitarget tracking with split and merged measurements. In Proc. IEEE Conf. Comp. Vision and Patt. Recog., pages 605-610, 2005. 2

[13] E. D. Miller, G. W. Fisher, L. E. Weiss, L. M. Walker, and P. G. Campbell. Dose-dependent cell growth in response to concentration modulated patterns of FGF-2 printed on fibrin. Biomaterials, 27:2213-2221, Apr. 2006. 1, 7

[14] D. P. Mukherjee, N. Ray, and S. T. Acton. Level set analysis for leukocyte detection and tracking. IEEE Trans. Image Processing, 13:562-572, Apr. 2004. 2

[15] S. Osher and J. A. Sethian. Fronts propagating with curvature dependent speed: Algorithms based on Hamilton-Jacobi formulations. Jour. Comp. Phys., 79:12-49, 1988. 2

[16] N. Otsu. A threshold selection method from gray level histograms. IEEE Trans. Syst., Man, Cybern., 9:62-66, Mar. 1979. 3

[17] D. Reynard, A. Wildenberg, A. Blake, and J. Marchant. Learning dynamics of complex motions from image sequences. In Proc. European Conf. Comp. Vision, pages $357-$ 568, Cambridge, UK, 1996. 4

[18] W. M. Saltzman. Tissue Engineering: Engineering Principles for the Design of Replacement Organs and Tissues. Oxford University Press, July 2004. 4

[19] F. Ségonne. Segmentation of Medical Images under Topological Constraints. PhD thesis, Dec. 2005. 2

[20] Y. Shi and W. C. Karl. Real-time tracking using level sets. In Proc. IEEE Conf. Comp. Vision and Patt. Recog., volume 2, pages 34-41, June 2005. 4

[21] S. Sternberg. Biomedical image processing. IEEE Computer, 16(1):22-34, Jan. 1983. 3

[22] L. E. Weiss, C. Amon, S. Finger, E. Miller, D. Romero, I. Verdinelli, L. Walker, and P. Campbell. Bayesian computer-aided experimental design of heterogeneous scaffolds for tissue engineering. Computer Aided Design, 37:1127-1139, 2005. 1, 6

[23] F. Yang, M. A. Mackey, F. Ianzini, G. Gallardo, and M. Sonka. Cell segmentation, tracking, and mitosis detection using temporal context. In J. S. Duncan and G. Gerig, editors, Proc. Medical Image Computing and Comp.-Assisted Intervention, volume I, pages 302-309, Oct. 2005. 2, 5

[24] C. Zimmer, E. Labruy 'ere, V. Meas-Yedid, N. Guill'en, and J.-C. Olivo-Marin. Segmentation and tracking of migrating cells in videomicroscopy with parametric active contours: A tool for cell-based drug testing. IEEE Trans. Med. Imag., 21(10):1212-1221, Oct. 2002. 2

[25] C. Zimmer and J.-C. Olivo-Marin. Coupled parametric active contours. IEEE Trans. Pattern Anal. Machine Intell., 27(11):1838-1842, Nov. 2005. 2 